\title{
'JOIN OUR CAMPAIGN TO SAFEGUARD THE DENTAL NURSE WORKFORCE'
}

\author{
BADN Presidential column by Jacqui Elsden
}

We

are halfway through the year and I am contemplating how the national roadmap in easing the restrictions will further affect our professional dental nursing lives.

Will we still be required to employ our enhanced PPE? What about the scheduling of appointments? Will we be able to return to pre-pandemic protocols and has there been recognition for us in our pay and conditions as a result of our continued contribution to the profession as we stepped up to the changes in our workplace, and our working hours during the global pandemic? We understand that dental practice is a small business and that overheads need to be met. However, dental nurse responsibility has moved on since the 1980s; we are professionals and should be acknowledged accordingly!

This is why we launched our campaign for the Recognition of Dental Nurses' Contribution to Dentistry.

It was clear during Lockdown 1.0 last year that many dental nurses were disappointed in the way they had been acknowledged by their employers. High call volumes were received at BADN's Head Office which portrayed worrying employment and financial problems for members and non-members.

Our 'Campaign' was launched to bring to the surface once again awareness regarding the valuable role that dental nurses have within dental teams across the UK. For many years now we have felt undervalued due to the low pay received, for a role considered 'the cornerstone' of many dental practices. Sadly, this was further experienced by some dental nurses as their employers refused to pay their dental nurses in full during the pandemic, leaving them with little or no income.

To make matters worse, those dental nurses who worked in NHS general dental practices (primary care) and then worked or volunteered for the urgent dental hubs or the Nightingale hospitals during Lockdown 1.0 , were not afforded the same privileges as other NHS employees, such as the one-hour supermarket slot devoted to NHS workers before supermarkets allowed in everyone else, because they did not have an NHS ID card. This also served to highlight, yet again, the fact that dental nurses who work in NHS primary care practices do not receive the same benefits as their secondary care dental nurse colleagues (such as the NHS pension scheme or NHS salary scales), but work equally as hard and are equally dedicated to their roles. Our campaign continues to focus on this inequality of the system.

$\mathrm{CPD}$ and GDC registration are further issues for dental nurses and part of our campaign. Not only do many dental nurses pay for their own CPD and GDC registration to be able to work in the UK, but in 2020, we were told that our ARF would not be reduced or waived and that we could not pay in instalments. Considering that many dental nurse registrants had not been at work for approximately a quarter of their registered year, it was a further blow and an additional reason for feeling undervalued by the profession. It was no surprise that many dental nurses did not re-register and left the profession because they simply could not afford to stay. Whilst the GDC have since introduced a direct debit scheme for the ARF on a quarterly basis, we at BADN still feel that this action has not gone far enough. Why should we pay the same fees as other DCPs who have higher salaries? Yet another inequality.

At least Key Worker status was eventually acknowledged for dental nurses, BUT this is just a small step in the 'acknowledgement arena' ... as far as I am concerned.

Dental nurses are registered dental care professionals and must be recognised as such - by employers, by colleagues and by the NHS. BADN are calling for all dental nurses in practice to be paid a salary which recognises not only their invaluable contribution to the dental team but also their training, knowledge and professionalism, as well as the financial outgoings required just to be allowed to work as a dental nurse - training costs, registration fees, indemnity cover and CPD costs - not just minimum wage.

By joining us in our campaign you will be supporting the work that we do to receive recognition of our contribution to dentistry, not just for all that you did for the profession and your patients in 2020, but for your future career and to safeguard the dental nurse workforce of the future. It will be interesting to see how many more dental nurses do not re-register in July this year because they have had enough of being undervalued by their colleagues.

If you are not yet a BADN member but wish to join your professional association and support our campaign, please visit www. badn.org.uk for more info and click on the yellow 'Join Now' ribbon in the top right hand corner.

\section{Healthwatch England has published a report ${ }^{1}$ on dentistry during the COVID-19 pandemic, revealing concerns around levels of access, which has been picked up in the national media. \\ The findings indicated that, between January and March 2021: \\ - $80 \%$ of people found it difficult to access timely care \\ - $59 \%$ reported a negative experience of care \\ - $3 \%$ reported a positive experience. \\ Reported problems concerning NHS \\ dentistry included delayed and unequal access, disrupted care, pressure to go private, being left in pain and provision of poor information. Responding to the findings, Healthwatch has called for action in four areas: \\ - A more rapid and radical reform of the way dentistry is commissioned and provided \\ - Using the reform of commissioning to tackle the twin crises of access and affordability}

HEALTHWATCH ENGLAND REPORT REVEALS CONCERNS AROUND DENTAL ACCESS

- Greater clarity in the information about NHS dentistry

- Look at using dental practices to support people’s general health.

\section{Reference}

1. Healthwatch. Dentistry during COVID-19 insight briefing. 24 May 2021. Available at: https://www.healthwatch.co.uk/ report/2021-05-24/dentistry-during-covid19-insight-briefing (accessed 28 May 2021). 